\title{
OPTICAL ASTROMETRY OF EXTRAGALACTIC RADIO SOURCES WITH THE TAUTENBURG SCHMIDT TELESCOPE
}

\author{
W.R. DICK \\ Zentralinstitut für Astrophysik \\ Rosa-Luxemburg-Strasse 17a \\ DDR-1561 Potsdam \\ Germany, Democratic Republic \\ I.I. KUMKOVA \\ Institute of Applied Astronomy \\ 8 Zhdanovskaya ul. \\ 197042 Leningrad \\ USSR
}

\begin{abstract}
Optical positions of objects from the IAU Commission 24 Working Group list of benchmark radio sources have been derived which will contribute to the link of the radio and optical reference frames. Results for 11 objects with an r.m.s. position error of 0.2 are presented and discussed.
\end{abstract}

\section{Introduction}

Since 1978, when a working group of IAU Commission 24 was established, a continuing effort has been made to obtain precise optical positions of extragalactic radio objects. Most of the published positions are for objects brighter than $18 \mathrm{mag}$, whereas the majority of recommended benchmark radio sources (Argue et al. 1984) have visual magnitudes beyond $18 \mathrm{mag}$. For statistics on the Northern sky see Dick and Kumkova (1989).

At the end of 1986 a program was started with the Tautenburg Schmidt Telescope with special emphasis on objects of 18 to 19 mag. The first measurements concentrated on those objects for which precise optical positions had already been published. Some details of the observations and measurements as well as a discussion of the first results were reported by Dick (1989). We used 50 to 80 reference stars from the AGK3 distributed over the whole plate.

\section{Results}

Table 1 presents results for the 11 objects. Each position is the mean of two values from a pair of plates. From the differences between these two values an "internal" standard error for the mean values of less than 0"15 (r.m.s.) was derived. A comparison with optical positions published by other authors shows that some of the derived coordinates seem to have systematic offsets from these values. The unweighted mean of the offsets is given in columns (5) and (6). Only those published positions with r.m.s. errors less than or equal to 0:2 (as given by the authors) have been taken into consideration. Their number is listed in column (7). 
For 8 objects a comparison is possible with the radio reference frame (Walter 1989). In most cases the residuals are identical with those calculated from a comparison with the compilation catalogue of Argue et al. (1984) at the 0."01 level. Neglecting the errors in the radio positions, which are considerably smaller than the errors in the optical positions, an "external" standard error of 0.2 in $\Delta \alpha \cos \delta$ and 0.3 in $\Delta \delta$ was derived. The mean offsets indicate that in the optical positions a systematic error is probably inherent due to a magnitude equation on Tautenburg Schmidt plates.

Some additional results ( $c f$. Dick 1990) which may be of general interest for photographic astrometry are published elsewhere.

Table 1. Optical positions of extragalactic radio sources

(1)

Source

$0106+013$

$0440-003$

$0552+398$

$0642+449$

$0736+017$

$0952+179$

$1055+018$

$1328+254$

$1442+101$

$1638+398$

$1641+399$
(2) $m_{\mathrm{v}}$

(3)

R.A. h m s

$(4)$
B1950.0) Dec.

164.496

18.5

18.5

$440 \quad 5.270$

$\begin{array}{lll}552 & 1.392\end{array}$

64252.984

19

73642.486

18

95211.822

$18.0 \quad 105555.288$

$18.0 \quad 132815.904$

$18.4 \quad 144250.460$

$18.5 \quad 163848.177$

16.3164117 .596
$+1191.27$

- 02321.02

+394822.11

+445430.90

$+1440.04$

$+175744.58$

$+1502.87$

$+252437.64$

+101111.96

+3952 29.22

+395410.36

\section{(5)} $\Delta \alpha \cos \delta$ (6) (7)

(8) $\Delta \delta \quad n \quad$ Epoch $\begin{array}{llll}+0.02 & -0.06 \quad 3 & 87.75\end{array}$ $\begin{array}{llll}+0.27 & -0.16 \quad 3 & 86.85\end{array}$

87.07
$+0.36-0.02-2 \quad 8.85$

$\begin{array}{llll}+0.36 & +0.02 \quad 2 & 86.85\end{array}$

$+0.34+0.00 \quad 6 \quad 87.07$

$\begin{array}{llll}-0.06 & +0.27 & 3 & 87.23\end{array}$

$+0.19+0.86 \quad 1 \quad 87.22$

$\begin{array}{llll}+0.33 & -0.24 & 1 & 87.30\end{array}$

$+0.36+0.18 \quad 3 \quad 87.22$

$\begin{array}{llll}-0.08 & +0.84 & 1 & 87.64\end{array}$

$+0.13 \quad+0.28 \quad 5 \quad 87.64$

\section{References}

Argue, A.N. et al. (1984) "A catalog of selected compact radio sources for the construction of an extragalactic radio/optical reference frame", Astron. Astrophys. 130, 191-199.

Dick, W.R. (1989) "Determining optical positions of benchmark radio sources with the Tautenburg Schmidt telescope (first results)", Mitt. Lohrmann-Obs. TU Dresden Nr. 56, 20-22.

Dick, W.R. (1990) "The astrometric properties of the Tautenburg Schmidt Telescope and its possible contributions to the realization of an inertial reference frame (Dissertation Abstract)", Astron. Nachr. 311, No. 2, in press.

Dick, W.R., and Kumkova, I.I. (1989) "The connection of the optical and radio reference systems by means of photographic astrometry", 6th Intern. Symp. Geodesy and Physics of the Earth, Proceed. Part I, Veröff. Zentralinst. Physik der Erde Potsdam No. 102, 70-74.

Walter, H.G. (1989) "A celestial reference frame based on extragalactic radio sources", Astron. Astrophys. Suppl. Ser. 79, 283-289.

\section{Discussion}

MoRRIson: Have you considered using reference stars from the Carlsberg Meridian Catalogues? W. Dick: When the CMC containing reference stars in the fields of extragalactic radio sources will be available, we will examine the possibility of using it. The question is whether the number of about 20 reference stars situated in the centre of the Schmidt plates is sufficient for modelling the plates. This has to be tested. 